\title{
Topology and combinatorics of real line arrangements
}

\author{
Enrique Artal Bartolo, Jorge Carmona Ruber, \\ José Ignacio Cogolludo Agustín and Miguel Marco Buzunáriz
}

\begin{abstract}
We prove the existence of complexified real arrangements with the same combinatorics but different embeddings in $\mathbb{P}^{2}$. Such a pair of arrangements has an additional property: they admit conjugated equations on the ring of polynomials over $\mathbb{Q}(\sqrt{5})$.
\end{abstract}

\section{Introduction}

In a famous preprint [Ryb98], Rybnikov proved the existence of two line arrangements $\mathcal{L}_{1}, \mathcal{L}_{2}$ in $\mathbb{P}^{2}:=\mathbb{C P}^{2}$ which have the same combinatorics but whose pairs, $\left(\mathbb{P}^{2}, \cup \mathcal{L}_{1}\right)$ and $\left(\mathbb{P}^{2}, \cup \mathcal{L}_{2}\right)$, are not homeomorphic. Unfortunately, this work has not been published in its final form. This may be due to the difficulty of verifying some of its statements. In another work [ACCM05], some parts of Rybnikov's paper have been presented and explained, including a detailed verification of the quoted result. Rybnikov also gave an outline for proving a stronger result: the fundamental groups of $\mathbb{P}^{2} \backslash \bigcup \mathcal{L}_{1}$ and $\mathbb{P}^{2} \backslash \bigcup \mathcal{L}_{2}$ are not isomorphic. Providing a complete proof of this result would be most interesting.

In the present work we are interested in the topology of complexified real arrangements. Complexified real arrangements form a very important class of arrangements where more topological properties are known. In this work we prove the existence of complexified real arrangements with the same combinatorics but different embeddings in $\mathbb{P}^{2}$. It is worth mentioning that Rybnikov's arrangements do not admit real equations. The counterexamples obtained in this work have an additional property: they admit conjugated equations on the ring of polynomials over a number field, namely $\mathbb{Q}(\sqrt{5})$. The goal of this paper is to show the existence of such a pair of arrangements. In the following outline of the paper, we stress the similarities and major differences with Rybnikov's approach.

(i) The first step towards finding such pairs of arrangements involves finding combinatorics whose moduli space is not connected. As a first approach, we will find ordered combinatorics whose moduli space is not connected (an easier task). We proceed by considering an ordered line arrangement with equations in a finite field and then studying its complex realizations. In Rybnikov's paper, the MacLane arrangement in $\mathbb{F}_{3} \mathbb{P}^{2}$ was considered.

(ii) In a second step we exhibit two ordered line arrangements $\mathcal{M}_{1}, \mathcal{M}_{2}$ which have the same ordered combinatorics but whose pairs, $\left(\mathbb{P}^{2}, \cup \mathcal{M}_{1}\right)$ and $\left(\mathbb{P}^{2}, \cup \mathcal{M}_{2}\right)$, are not homeomorphic by an order-preserving map. In order to prove this result we need to obtain some non-generic braid monodromies. In Rybnikov's paper, the author constructs an invariant of the isomorphism class of fundamental groups of the complements of lines whose ordered combinatorics induce

Received 24 September 2003, accepted in final form 23 October 2004.

2000 Mathematics Subject Classification 32S22, 14N20, 20F36 (primary), 20E18, 32S50, 57M05 (secondary).

Keywords: line arrangements, braid monodromy.

The first, third and fourth authors are partially supported by BFM2001-1488-C02-02; the second author is partially supported by BFM2001-1488-C02-01.

This journal is (C) Foundation Compositio Mathematica 2005. 


\section{TOPOLOGY AND COMBINATORICS OF REAL LINE ARRANGEMENTS}

the identity on the first homology group. In [ACCM05] we describe an extension of Rybnikov's invariant which has been implemented using Maple. Using it, we are able to double check Rybnikov's example. Unfortunately this invariant does not give any valuable information in our example.

(iii) Finally, using the aforementioned arrangements $\mathcal{M}_{1}, \mathcal{M}_{2}$ we construct a pair of arrangements $\mathcal{L}_{1}, \mathcal{L}_{2}$ so that the pairs $\left(\mathbb{P}^{2}, \bigcup \mathcal{L}_{1}\right)$ and $\left(\mathbb{P}^{2}, \cup \mathcal{L}_{2}\right)$ are not homeomorphic. This idea is part of Rybnikov's strategy: the fact that $\mathcal{M}_{1}, \mathcal{M}_{2}$ are real arrangements allows us to use simpler arguments.

Note that in our case the fundamental groups of $\mathbb{P}^{2} \backslash \bigcup \mathcal{L}_{1}$ and $\mathbb{P}^{2} \backslash \bigcup \mathcal{L}_{2}$ have isomorphic profinite completions, but we do not know whether or not they are isomorphic.

\section{An arrangement over a finite field}

In what follows, a brief description of combinatorics and ordered combinatorics of a line arrangement are given.

Definition 2.1. A line combinatorics (projective configuration in [Ryb98]) is a triple $(\mathcal{L}, \mathcal{P}, \in)$ where $\mathcal{L}$ and $\mathcal{P}$ are finite subsets and $\in$ is a relation between $\mathcal{P}$ and $\mathcal{L}$ satisfying:

(i) for all $\ell, \ell^{\prime} \in \mathcal{L}, \ell \neq \ell^{\prime}, \exists ! p \in \mathcal{P}$ such that $p \in \ell$ and $p \in \ell^{\prime}$ (in notation $p=\ell \cap \ell^{\prime}$ );

(ii) for all $p \in \mathcal{P} \exists \ell, \ell^{\prime} \in \mathcal{L}, \ell \neq \ell^{\prime}$, such that $p=\ell \cap \ell^{\prime}$.

Analogously we define an ordered line combinatorics, where the elements of $\mathcal{L}=\left\{\ell_{0}, \ell_{1}, \ldots, \ell_{n}\right\}$ are ordered. If the context is not ambiguous, we will omit both $\mathcal{P}$ and $\in$. The cardinal $\#\{\ell \mid p \in \ell\}$ is denoted as $\nu(p)$ and referred to as the multiplicity of $p$ (as usual, points of multiplicity 2 are also called double points and points of multiplicity 3 triple points). The second condition in a line combinatorics $(\mathcal{L}, \mathcal{P}, \in)$ implies that $\nu(p) \geqslant 2$ for all $p \in \mathcal{P}$.

A simple way to obtain line combinatorics is via arrangements in $\mathbb{F}_{q} \mathbb{P}^{2}$ for some finite field $\mathbb{F}_{q}$. In such cases it is more practical to consider arrangements of points and then dualize.

For example, the starting point in [Ryb98] is the MacLane arrangement. This arrangement can be defined as follows. Consider $\mathbb{F}_{3} \mathbb{P}^{2}$ as the union of $\mathbb{F}_{3}^{2}$ and the line at infinity. Consider also the points of $\mathbb{F}_{3}^{2} \backslash\{0\}$ : the MacLane combinatorics is the abstract line combinatorics corresponding to the dual of this eight-point arrangement.

Definition 2.2. Let $\mathcal{L}$ be a line combinatorics. An automorphism of $\mathcal{L}$ is a permutation of $\mathcal{L}$ preserving the incidence relations. The set of such automorphisms is the automorphism group of $\mathcal{L}$.

Example 2.3. It is easily seen that the automorphism group of MacLane combinatorics is naturally isomorphic to $\mathrm{GL}\left(2 ; \mathbb{F}_{3}\right)$.

The starting point of this work is a similar idea. Let us consider $\mathbb{F}_{4} \mathbb{P}^{2}$ as the union of $\mathbb{F}_{4}^{2}$ and the line at infinity $L_{\infty}$. Let us consider

$$
A:=\left(\begin{array}{cc}
0 & 1 \\
1 & \zeta
\end{array}\right) \in \mathrm{GL}\left(2 ; \mathbb{F}_{4}\right),
$$

where $\zeta$ is any of the elements of $\mathbb{F}_{4} \backslash \mathbb{F}_{2}$ (and hence the other is $\bar{\zeta}=1+\zeta=\zeta^{-1}=\zeta^{2}$ ). Note that $A^{5}=I_{2}$. The set

$$
\mathcal{B}:=L_{\infty} \cup\left\{A^{j}\left(\begin{array}{l}
1 \\
0
\end{array}\right) \mid 0 \leqslant j \leqslant 4\right\}=L_{\infty} \cup\left\{\left(\begin{array}{l}
1 \\
0
\end{array}\right),\left(\begin{array}{l}
0 \\
1
\end{array}\right),\left(\begin{array}{l}
1 \\
\zeta
\end{array}\right),\left(\begin{array}{l}
\zeta \\
\zeta
\end{array}\right),\left(\begin{array}{l}
\zeta \\
1
\end{array}\right)\right\}
$$




\section{E. Artal Bartolo et al.}

contains ten points, the last five of which will be denoted by $P_{1}, \ldots, P_{5}$. Note that each point lies in a different line through the origin. Let us denote by $Q_{j}$ the point at infinity of the line passing through both the origin and $P_{j}, j=1, \ldots, 5$. The following is a list of all the lines of $\mathbb{F}_{4} \mathbb{P}^{2}$ that contain at least two points (after each equation, a list of points in $\mathcal{B}$ contained in the line is provided):

(i) the line $L_{\infty}$ containing $Q_{1}, \ldots, Q_{5}$;

(ii) lines through $Q_{1}: y=0\left(P_{1}\right), y=z\left(P_{2}, P_{5}\right), y=\zeta z\left(P_{3}, P_{4}\right)$;

(iii) lines through $Q_{2}: x=z\left(P_{1}, P_{3}\right), x=0\left(P_{2}\right), x=\zeta z\left(P_{4}, P_{5}\right)$;

(iv) lines through $Q_{3}: y=\zeta(x+z)\left(P_{1}, P_{5}\right), y=\zeta x+z\left(P_{2}, P_{4}\right), y=\zeta x\left(P_{3}\right)$;

(v) lines through $Q_{4}: y=x+z\left(P_{1}, P_{2}\right), y=x+\bar{\zeta} z\left(P_{3}, P_{5}\right), y=x\left(P_{4}\right)$;

(vi) lines through $Q_{5}: y=\bar{\zeta}(x+z)\left(P_{1}, P_{4}\right), y=\bar{\zeta} x+z\left(P_{2}, P_{3}\right), y=\zeta x\left(P_{5}\right)$.

Note that each pair $P_{i}, P_{j}, 1 \leqslant i<j \leqslant 5$ appears exactly once in the above list. The line combinatorics $\mathcal{C}$ we are interested in is dual to $\mathcal{B}$. It contains the following ten lines:

$L_{1}, \ldots, L_{5}$ dual to $P_{1}, \ldots, P_{5}$ respectively and

$M_{1}, \ldots, M_{5}$ dual to $Q_{1}, \ldots, Q_{5}$ respectively.

The intersection points of these lines are:

$O:=M_{1} \cap \cdots \cap M_{5}$, dual to the origin $\left(\begin{array}{l}0 \\ 0\end{array}\right)$;

$P_{1,\{2,5\}}, P_{1,\{3,4\}}, P_{2,\{1,3\}}, P_{2,\{4,5\}}, P_{3,\{1,5\}}, P_{3,\{2,4\}}, P_{4,\{1,2\}}, P_{4,\{3,5\}}, P_{5,\{1,4\}}, P_{5,\{2,3\}}$, where

$P_{i,\{j, k\}}:=M_{i} \cap L_{j} \cap L_{k}$;

$R_{i}:=M_{i} \cap L_{i}, i=1, \ldots, 5$, dual to the line passing through the origin, $P_{i}$ and $Q_{i}$.

Note that the Falk-Sturmfels arrangement (see [CS97]) is the result of deleting one of the $M_{i}$ lines from $\mathcal{C}$.

Lemma 2.4. Let $\psi \in \operatorname{Aut}(\mathcal{C})$. Then there exists a unique $\sigma \in \Sigma_{5}$ such that $\psi\left(L_{i}\right)=L_{i^{\sigma}}$ and $\psi\left(M_{i}\right)=M_{i^{\sigma}}, i=1, \ldots, r$. The mapping $\psi \mapsto \sigma$ defines a monomorphism $\operatorname{Aut}(\mathcal{C}) \rightarrow \Sigma_{5}$ which will be identified with the inclusion.

Proof. First of all note that any $\psi \in \operatorname{Aut}(\mathcal{C})$ preserves the multiplicity of any point. Since $\{O\}=$ $\{P \in \mathcal{C} \mid \nu(P)=5\}$ one concludes that $\psi$ fixes $O$ and hence should interchange the five lines $M_{1}, \ldots, M_{5}$ containing $O$. Let us denote by $\sigma \in \Sigma_{5}$ the permutation satisfying $\psi\left(M_{i}\right)=M_{i^{\sigma}}$. Note that each line $M_{i}$ determines $R_{i}$ (as the only point of multiplicity 2 contained in $M_{i}$ ) and hence it determines $L_{i}$ (as the other line containing $R_{i}$ ). Therefore, $\psi\left(R_{i}\right)=R_{i^{\sigma}}$ and $\psi\left(L_{i}\right)=L_{i^{\sigma}}$.

Remark 2.5. Because of this identification $\operatorname{Aut}(\mathcal{C})$ can be considered to act on $\mathcal{C}$ on the right.

Example 2.6. The action of the matrix $A$ induces an automorphism $\psi_{A}$ of $\mathcal{C}$ given by the 5-cycle $(1,2,3,4,5)$.

Example 2.7. Let us consider the automorphism of $\mathbb{F}_{4}^{2}$ defined by $B \sigma$ where

$$
B:=\left(\begin{array}{cc}
1 & \zeta \\
0 & \zeta
\end{array}\right) \in \mathrm{GL}\left(2 ; \mathbb{F}_{4}\right)
$$

and $\sigma$ is the Galois involution of $\mathbb{F}_{4} \mid \mathbb{F}_{2}$. The projective completion of this mapping preserves $\mathcal{B}$ and induces an automorphism $\bar{\psi}_{B}$ of $\mathcal{C}$ determined by the permutation $(2,4,5,3)$.

Remark 2.8. Regarding again $\operatorname{Aut}(\mathcal{C})$ as a subgroup of $\Sigma_{5}$, it is easily seen that $\bar{\psi}_{B}^{-1} \psi_{A} \bar{\psi}_{B}=\psi_{A}^{3}$, that is, $\left\langle\psi_{A}\right\rangle$ is a normal subgroup in $G:=\left\langle\psi_{A}, \bar{\psi}_{B}\right\rangle$. Since $G /\left\langle\psi_{A}\right\rangle \cong\left\langle\bar{\psi}_{B}\right\rangle$, the subgroup $G$ is a semidirect product of $\left\langle\psi_{A}\right\rangle \cong \mathbb{Z}_{5}$ and $\left\langle\bar{\psi}_{B}\right\rangle \cong \mathbb{Z}_{4}$ and hence has order 20 . 
Lemma 2.9. Under the above considerations $\operatorname{Aut}(\mathcal{C})=G$.

Proof. It is enough to check that $\operatorname{Aut}(\mathcal{C}) \subset G$. For the sake of simplicity, we will denote an automorphism in $\operatorname{Aut}(\mathcal{C})$ and its induced permutation in $\Sigma_{5}$ by the same symbol.

Let us consider $\psi$ an automorphism fixing at least two lines of type $M_{i}$. After conjugation by an element in $G$, one may assume that these lines are $M_{1}, M_{2}$. Hence $L_{1}$ and $L_{2}$ are also fixed (Lemma 2.4). Using $\psi\left(P_{1,\{2,5\}}\right)=P_{1,\left\{2,5^{\psi}\right\}}, \psi\left(P_{4,\{1,2\}}\right)=P_{4^{\psi},\{1,2\}}$, and $\psi\left(P_{2,\{1,3\}}\right)=P_{2,\left\{1,3^{\psi}\right\}}$, the lines $L_{5}, L_{4}$, and $L_{3}$ are also respectively fixed, hence $\psi$ is the identity.

Let us consider $\psi$ an automorphism fixing only one line of type $M_{i}$. Again, after conjugation by an element in $G$ one may suppose such a line is $M_{1}$. Since $\bar{\psi}_{B}$ fixes $M_{1}$ and permutes the others, after multiplying $\psi$ by a certain power of $\bar{\psi}_{B}$ one may suppose that $M_{2}$ is also fixed; then by the previous step $\psi \in G$.

Finally, let us consider a general $\psi$. Since $\psi_{A}$ is transitive, after multiplying $\psi$ by a certain power of $\psi_{A}$ one may suppose that $M_{1}$ is fixed; then by the previous step $\psi \in G$.

Remark 2.10. Following the notation of the previous examples, the automorphisms $\psi_{M}$ defined by a linear transformation $M$ will be called positive, whereas the automorphisms $\bar{\psi}_{M}$ defined by $M \sigma$ ( $\sigma$ being the Galois transformation of $\mathbb{F}_{4} \mid \mathbb{F}_{2}$ ) will be called negative. When the automorphisms are viewed as permutations, the concepts of positive and negative agree with their signature.

\section{Complex realization}

Definition 3.1. Let $\mathcal{C}$ be a line combinatorics. An arrangement $\mathcal{L}$ of $\mathbb{P}^{2}=\mathbb{C P}^{2}$ is a complex realization of $\mathcal{C}$ if its combinatorics agrees with $\mathcal{C}$. An ordered complex realization of an ordered line combinatorics is defined accordingly.

Notation 3.2. The space of all complex realizations of a line combinatorics $\mathcal{C}$ is denoted by $\Sigma(\mathcal{C})$. This is a quasiprojective subvariety of $\mathbb{P}^{n(n+3) / 2}$, where $n:=\# \mathcal{C}$. If $\mathcal{C}$ is ordered, we denote by $\Sigma^{\text {ord }}(\mathcal{C}) \subset\left(\check{\mathbb{P}}^{2}\right)^{n}$ the space of all ordered complex realizations of $\mathcal{C}$.

There is a natural action of $\operatorname{PGL}(3 ; \mathbb{C})$ on such spaces. This justifies the following definition.

Definition 3.3. The moduli space of a combinatorics $\mathcal{C}$ is the quotient $\mathcal{M}(\mathcal{C}):=\Sigma(\mathcal{C}) / \operatorname{PGL}(3 ; \mathbb{C})$. The ordered moduli space $\mathcal{M}^{\text {ord }}(\mathcal{C})$ of an ordered combinatorics $\mathcal{C}$ is defined accordingly.

Example 3.4. Let us consider the MacLane line combinatorics $\mathcal{N}$. It is well known that $\# \mathcal{M}(\mathcal{N})=1$ and that $\# \mathcal{M}^{\text {ord }}(\mathcal{N})=2$. One can find representatives having equations in the polynomial ring over the field of cubic roots of unity. Moreover, the MacLane line combinatorics has no real realization.

Remark 3.5. Let us consider an ordered line combinatorics $\mathcal{C}$. It is easily seen that $\operatorname{Aut}(\mathcal{C})$ acts on both $\mathcal{M}(\mathcal{C})$ and $\mathcal{M}^{\text {ord }}(\mathcal{C})$.

Example 3.6. The action of $\operatorname{Aut}(\mathcal{N}) \cong \mathrm{GL}\left(2, \mathbb{F}_{3}\right)$ on the moduli spaces is as follows: matrices of determinant +1 (respectively -1 ) fix (respectively interchange) the two elements of $\mathcal{M}^{\text {ord }}(\mathcal{N})$. Of course complex conjugation also acts on $\mathcal{M}^{\text {ord }}(\mathcal{N})$ interchanging the two elements. From the topological point of view, after fixing two representatives $\mathcal{N}^{ \pm}$of $\mathcal{N}$ one has that:

(i) there exists a homeomorphism $\left(\mathbb{P}^{2}, \cup \mathcal{N}^{+}\right) \rightarrow\left(\mathbb{P}^{2}, \cup \mathcal{N}^{-}\right)$preserving orientations on both $\mathbb{P}^{2}$ and the lines - such a homeomorphism does not respect the ordering;

(ii) there exists a homeomorphism $\left(\mathbb{P}^{2}, \cup \mathcal{N}^{+}\right) \rightarrow\left(\mathbb{P}^{2}, \cup \mathcal{N}^{-}\right)$preserving orientations on $\mathbb{P}^{2}$, but not on the lines - such a homeomorphism respects the ordering. 


\section{E. Artal Bartolo et al.}

In what follows, we study the space and moduli of complex realizations for the combinatorics $\mathcal{C}$ introduced in $\S 2$. Let us fix an ordering in the combinatorics so as to choose coordinates. Let us suppose that a complex realization exists. Up to the action of $\operatorname{PGL}(3 ; \mathbb{C})$ one may assume that

$$
M_{1}: z=0, \quad M_{2}: x=0, \quad M_{3}: x=z, \quad M_{4}: x=\alpha z, \quad M_{5}: x=\beta z
$$

where $\alpha, \beta \in \mathbb{C} \backslash\{0,1\}, \alpha \neq \beta$ (note that $\alpha, \beta$ cannot be fixed a priori since they represent crossratios, which are projective invariants). Taking the incidence relations into account and by means of the subgroup of projective transformations which fix $M_{1}, M_{2}$, and $M_{3}$, one can also assume that $L_{5}: y=0$ and $L_{4}: y=z$. Note that the only projective transformation which fixes $M_{1}, M_{2}, M_{3}$, $L_{4}$, and $L_{5}$ is the identity. Also note the following.

(i) $L_{1}$ passes through $L_{5} \cap M_{2}=[0: 0: 1]$ and $L_{4} \cap M_{3}=[1: 1: 1]$, hence $L_{1}: x=y$.

(ii) $L_{2}$ passes through $L_{4} \cap M_{5}=[\beta: 1: 1], L_{5} \cap M_{3}=[1: 0: 1]$, and $L_{1} \cap M_{4}=[\alpha: \alpha: 1]$, hence

$$
L_{2}: \alpha x-(\alpha-1) y-\alpha z=0, \quad \beta=\frac{2 \alpha-1}{\alpha} .
$$

(iii) $L_{3}$ passes through $L_{1} \cap M_{5}=[2 \alpha-1: 2 \alpha-1: \alpha], L_{4} \cap M_{2}=[0: 1: 1], L_{5} \cap M_{4}=[\alpha: 0: 1]$, and $L_{2} \cap M_{1}=[\alpha-1: \alpha: 0]$. In principle one should obtain four equations out of these conditions, but only three of them are independent, yielding

$$
L_{3}: x+\alpha y-\alpha z=0, \quad \alpha^{2}+\alpha-1=0 .
$$

The family $\mathcal{M}^{\text {ord }}(\mathcal{C})$ depends on one parameter $\alpha$ satisfying the above equation. The other root of this equation is $\gamma:=-1-\alpha$, which also verifies $\gamma^{2}+\gamma-1=0$, will be taken as the parameter. Summarizing, one has the following.

Proposition 3.7. The space $\mathcal{M}^{\text {ord }}(\mathcal{C})$ has two elements. Moreover, representatives can be chosen to have the following equations:

$$
\begin{gathered}
M_{1}: z=0, \quad M_{2}: x=0, \quad M_{3}: x=z, \quad M_{4}: x=-(\gamma+1) z, \quad M_{5}: x=(\gamma+2) z, \\
L_{1}: y=x, \quad L_{2}: y=\gamma(x-z), \quad L_{3}: y=\gamma x+z, \quad L_{4}: y=z, \quad L_{5}: y=0,
\end{gathered}
$$

where $\gamma^{2}+\gamma-1=0$.

Remark 3.8. The previous proposition implies that there are representatives of the two ordered complex realizations of $\mathcal{C}$ having real equations conjugated in the ring of polynomials over the number field $\mathbb{Q}(\sqrt{5})$. It is easily seen that the positive (respectively negative) automorphisms of $\mathcal{C}$ preserve (respectively interchange) the elements in $\mathcal{M}^{\text {ord }}(\mathcal{C})$. In particular, $\# \mathcal{M}(\mathcal{C})=1$.

\section{Topology of the realizations}

The first main result of Rybnikov's work [Ryb98] reads as follows: let us denote by $\mathcal{N}^{ \pm}$two representatives of the elements of $\mathcal{M}^{\text {ord }}(\mathcal{N})$. Then there is no isomorphism of the fundamental groups of $\mathbb{P}^{2} \backslash \cup \mathcal{N}^{+}$and $\mathbb{P}^{2} \backslash \cup \mathcal{N}^{-}$inducing the identity in the homology groups. In particular, there is no orientation-preserving homeomorphism between $\left(\mathbb{P}^{2}, \cup \mathcal{N}^{+}\right)$and $\left(\mathbb{P}^{2}, \cup \mathcal{N}^{-}\right)$.

Analogously, one can consider the two ordered arrangements $\mathcal{C}^{ \pm}$representing the elements of $\mathcal{M}^{\text {ord }}(\mathcal{C})$. They are determined by the equations given in Proposition 3.7 and by the choice of $\gamma^{ \pm}:=$ $(-1 \pm \sqrt{5}) / 2$. Let us denote by $L_{i}^{ \pm}$and $M_{i}^{ \pm}, i=1, \ldots, 5$ the lines as in $\S 2$. Affine pictures are shown in Figures 1 and 2, where $M_{1}^{ \pm}$are the corresponding lines at infinity.

As pointed out in the introduction, we have completely checked Rybnikov's computations concerning MacLane arrangements [ACCM05]. Rybnikov's approach does not work with $\mathcal{C}$ and we do not know whether or not there exists an isomorphism of the fundamental groups of $\mathbb{P}^{2} \backslash \bigcup \mathcal{C}^{+}$and $\mathbb{P}^{2} \backslash \cup \mathcal{C}^{-}$inducing the identity in the homology groups. Nevertheless, we are able to prove that there 


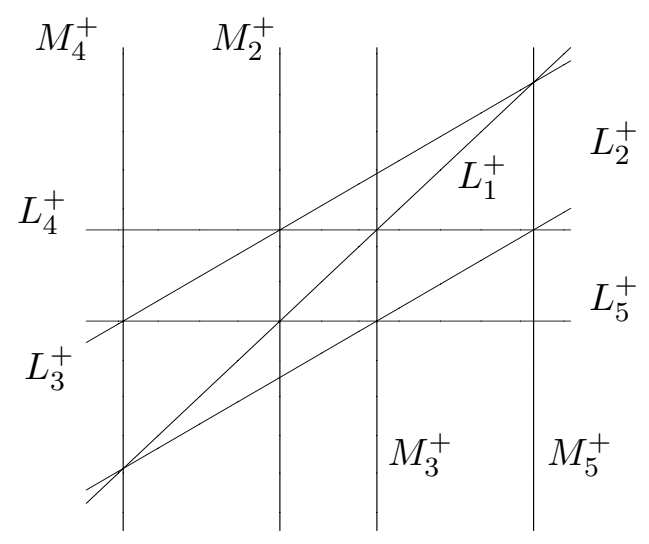

FiguRE 1. Arrangement $\mathcal{C}^{+}$.

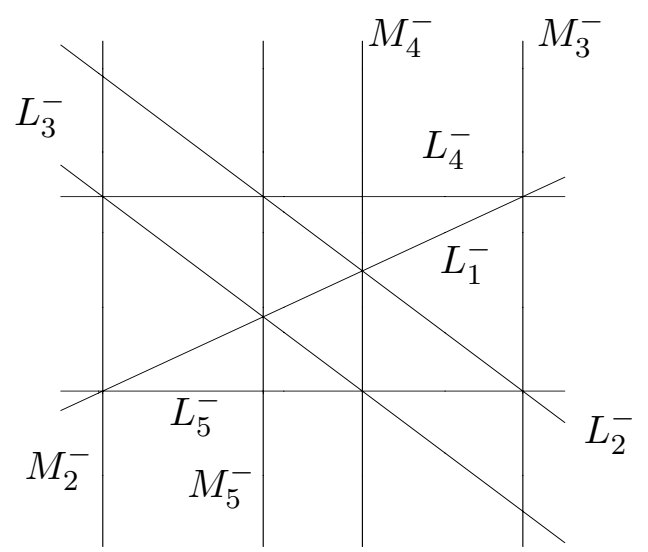

Figure 2. Arrangement $\mathcal{C}^{-}$.

is no orientation-preserving homeomorphism $\left(\mathbb{P}^{2}, \cup \mathcal{C}^{+}\right) \rightarrow\left(\mathbb{P}^{2}, \cup \mathcal{C}^{-}\right)$. The required invariant is a non-generic braid monodromy. Let us introduce some concepts in order to adapt braid monodromy to line arrangements.

Definition 4.1. An affine horizontal arrangement $\mathcal{L}$ is an affine line arrangement in $\mathbb{C}^{2}$ with no vertical lines. The ramification set $A_{\mathcal{L}} \subset \mathbb{C}$ is the set of $x$-coordinates of the multiple points of $\mathcal{L}$.

DeFinition 4.2. An ordered affine horizontal arrangement $\mathcal{L}$ is an affine horizontal arrangement with an ordering on both $\mathcal{L}$ and $A_{\mathcal{L}}$. By means of the usual embedding $\mathbb{C}^{2} \subset \mathbb{P}^{2}$, the ordered fibered projective arrangement $\mathcal{L}^{\varphi}$ associated with $\mathcal{L}$ is obtained by considering:

(i) the projectivization of the lines in $\mathcal{L}$;

(ii) the line at infinity $L_{\infty}$;

(iii) the projectivization of the fibers of $A_{\mathcal{L}}$ (the vertical lines, pre-images of $A_{\mathcal{L}}$ ).

Example 4.3. The non-vertical lines in Figures 1 and 2 give two affine horizontal arrangements $\mathcal{L}^{ \pm}$. With a suitable order, their ordered fibered projective arrangements are $\mathcal{C}^{ \pm}$.

Remark 4.4. Given an ordered affine horizontal arrangement $\mathcal{L}$, the mapping $\mathbb{P}^{2} \backslash \mathcal{L}^{\varphi} \rightarrow \mathbb{C} \backslash A_{\mathcal{L}}$, induced by the projection $(x, y) \mapsto x$ is a locally trivial fibration. 


\section{E. Artal Bartolo et al.}

Definition 4.5. Let $Z$ be a connected projective manifold and let $H$ be a hypersurface of $Z$. Let $* \in Z \backslash H$ and let $K$ be an irreducible component of $H$. A homotopy class $\gamma \in \pi_{1}(Z \backslash H$; $*)$ is called a meridian about $K$ with respect to $H$ if $\gamma$ has a representative $\delta$ satisfying the following properties:

(i) there is a smooth complex analytic disk $\Delta \subset Z$ transverse to $H$ such that $\Delta \cap H=\left\{*^{\prime}\right\} \subset K$ (transversality implies that $*^{\prime}$ is a smooth point of $H$ );

(ii) there is a path $\alpha$ in $Z \backslash H$ from $*$ to $*^{\prime \prime} \in \partial \Delta$;

(iii) $\delta=\alpha \cdot \beta \cdot \alpha^{-1}$, where $\beta$ is the closed path obtained by traveling from $*^{\prime \prime}$ along $\partial \Delta$ in the positive direction.

Definition 4.6. Let $A:=\left\{a_{1}, \ldots, a_{r}\right\} \subset \mathbb{C}$ and let $\Delta \subset \mathbb{C}$ be a disk containing $A$ in its interior. Fix $* \in \partial \Delta$. A geometric basis of the free group $\pi_{1}(\mathbb{C} \backslash A ; *)$ is a basis $\gamma_{1}, \ldots, \gamma_{r}$ where each $\gamma_{i}$ is a meridian of a point in $A$ and $\gamma_{r} \cdots \gamma_{1}=\partial \Delta$ positively oriented.

Remark 4.7. In order to compute braid monodromies we use the reversed lexicographic order on $\mathbb{C}$, i.e. if $z, w \in \mathbb{C}, z \neq w$, then $z \succ w$ if $\Re z>\Re w$ or $\Re z=\Re w$ and $\Im z>\Im w$.

Construction 4.8. Let us construct a special geometric basis of $\pi_{1}(\mathbb{C} \backslash A ; *)$, where $*$ is a large enough real number and $A:=\left\{a_{1}, \ldots, a_{r}\right\} \subset \mathbb{C}$. Let us order the elements of $A$ such that $a_{1} \succ$ $\cdots \succ a_{r}$. Consider the polygonal path $\Gamma$ with vertices $*, a_{1}, \ldots, a_{r}$. Let us choose a small enough $\varepsilon>0$ so that the disks $\Delta_{i}$ of radius $\varepsilon$ centered at $a_{1}, \ldots, a_{r}$ are pairwise disjoint. The geometric basis $\gamma_{1}, \ldots, \gamma_{r}$ is defined as follows. Fix $i \in\{1, \ldots, r\}$; let us consider $\alpha_{i}$ a path starting at $*$ and running along $\Gamma$ outside the interior of the disks $\Delta_{1}, \ldots, \Delta_{i-1}$ and counterclockwise along the arcs $\partial \Delta_{1}, \ldots, \partial \Delta_{i-1}$. This path should stop at the first intersection $*_{i}$ of $\Gamma$ with $\partial \Delta_{i}$. Let us denote by $\delta_{i}$ the loop based at $*_{i}$ and going counterclockwise along $\partial \Delta_{i}$. Then $\gamma_{i}:=\alpha_{i} \cdot \delta_{i} \cdot \alpha_{i}^{-}$. This basis will be called a lexicographic basis.

Example 4.9. Let us denote by $\alpha_{5}^{+}, \alpha_{3}^{+}, \alpha_{2}^{+}$, and $\alpha_{4}^{+}$a lexicographic geometric basis of $\pi_{1}\left(\mathbb{C} \backslash A_{\mathcal{L}^{+}} ; R\right)$, $R \gg 0$; the indices of $\alpha_{i}^{+}$refer to the lines they are meridians of. Analogously, $\alpha_{3}^{-}, \alpha_{4}^{-}, \alpha_{5}^{-}$, and $\alpha_{2}^{-}$will denote a lexicographic geometric basis of $\pi_{1}\left(\mathbb{C} \backslash A_{\mathcal{L}^{-}} ; R\right)$. Let us recall that $A_{\mathcal{L}^{ \pm}}=$ $\left\{\gamma^{ \pm}+2,1,0,-\left(\gamma^{ \pm}+1\right)\right\}$.

Let $\mathbb{B}_{n}$ be the abstract braid group on $n=\# \mathcal{C}$ strings given by the standard presentation:

$$
\left\langle\sigma_{1}, \ldots, \sigma_{n-1}:\left[\sigma_{i}, \sigma_{j}\right]=1,|i-j| \geqslant 2, \sigma_{i} \sigma_{i+1} \sigma_{i}=\sigma_{i+1} \sigma_{i} \sigma_{i+1}, i=1, \ldots, n-2\right\rangle .
$$

The pure braid group $\mathbb{P}_{n}$ is the kernel of the natural epimorphism of $\mathbb{B}_{n}$ onto the symmetric group $\Sigma_{n}$ (obtained from the above presentation by adding the relations $\sigma_{i}^{2}=1$ ).

Let $\mathbf{y} \subset \mathbb{C}$ be a subset of exactly $n$ elements. Let us recall that the braid group $\mathbb{B}_{\mathbf{y}}$ is the set of homotopy classes, relative to $\{0,1\}$, of sets of paths $\left\{\gamma_{1}, \ldots, \gamma_{n}\right\}, \gamma_{j}:[0,1] \rightarrow \mathbb{C}$, starting and ending at $\mathbf{y}$ and such that for all $t \in[0,1],\left\{\gamma_{1}(t), \ldots, \gamma_{n}(t)\right\}$ is a set of $n$ distinct points. The elements of $\mathbb{B}_{\mathbf{y}}$ are called braids based at $\mathbf{y}$ and are represented as a set of non-intersecting paths in $\mathbb{C} \times[0,1]$, as usual. The pure braids correspond to sets of loops and they form the subgroup $\mathbb{P}_{\mathbf{y}}$. Products are also defined in the standard way. Analogously, if $\mathbf{y}_{1}, \mathbf{y}_{2} \subset \mathbb{C}$ are subsets of exactly $n$ elements then $\mathbb{B}_{\mathbf{y}_{2}, \mathbf{y}_{1}}$ is the set of braids starting at $\mathbf{y}_{1}$ and ending at $\mathbf{y}_{2}$. The abstract braid group is canonically identified with $\mathbb{B}_{\mathbf{y}_{0}}$, where $\mathbf{y}_{0}:=\{-1, \ldots,-n\}$. The groups $\mathbb{P}_{\mathbf{y}_{0}}$ and $\mathbb{P}_{n}$ are identified as well.

Example 4.10. There is a special element of $\mathbb{B}_{\mathbf{y}_{2}, \mathbf{y}_{1}}$ which is called the $\left(\mathbf{y}_{2}, \mathbf{y}_{1}\right)$-lexicographic braid. It is easily defined as follows. Let us order $\mathbf{y}_{1}$ and $\mathbf{y}_{2}$ according to the lexicographic order. The braid is defined by taking the linear segments joining the corresponding elements in $\mathbf{y}_{1}$ and $\mathbf{y}_{2}$. The main point of this construction is that given $\mathbf{y}_{1}$ and $\mathbf{y}_{2}$ one can identify $\mathbb{B}_{\mathbf{y}_{2}, \mathbf{y}_{1}}$ with $\mathbb{B}_{n}$ by means of the lexicographic braids associated with $\left(\mathbf{y}_{0}, \mathbf{y}_{1}\right)$ and $\left(\mathbf{y}_{2}, \mathbf{y}_{0}\right)$. 


\section{TOPOLOGY AND COMBINATORICS OF REAL LINE ARRANGEMENTS}

The braid monodromy of an ordered affine horizontal arrangement $\mathcal{L}$ can be defined as follows. Let us choose $* \in \mathbb{C} \backslash A_{\mathcal{L}}$ and let $\mathbf{y}^{*} \subset \mathbb{C}$ the set of $y$-coordinates of the points in $\mathcal{L}$ with $x$-coordinate $*$. Moving around a loop in $\mathbb{C} \backslash A_{\mathcal{L}}$ based at $*$ one can obtain a braid in $\mathbb{B}_{\mathbf{y}^{*}}$ and conjugating by the $\left(\mathbf{y}_{0}, \mathbf{y}^{*}\right)$-lexicographic braid one obtains a braid in $\mathbb{P}_{n}$.

DeFinition 4.11. The braid monodromy of an ordered affine horizontal arrangement $\mathcal{L}$ is the group homomorphism $\rho: \pi_{1}\left(\mathbb{C} \backslash A_{\mathcal{L}} ; *\right) \rightarrow \mathbb{P}_{n}$ defined above.

Remark 4.12. The braid monodromy is well defined up to conjugation in $\mathbb{P}_{n}$.

Proposition 4.13. The braid monodromies $\rho^{ \pm}$of the arrangements $\mathcal{L}^{ \pm}$are given by

$$
\begin{aligned}
& \rho^{+}\left(\alpha_{5}^{+}\right)=\rho^{-}\left(\alpha_{3}^{-}\right)=\sigma_{1}^{2} \sigma_{3}^{2} \\
& \rho^{+}\left(\alpha_{3}^{+}\right)=\rho^{-}\left(\alpha_{4}^{-}\right)=\left(\sigma_{1} \sigma_{3}\right) *\left(\sigma_{2}^{2} \sigma_{4}^{2}\right) \\
& \rho^{+}\left(\alpha_{2}^{+}\right)=\rho^{-}\left(\alpha_{5}^{-}\right)=\left(\sigma_{1} \sigma_{3} \sigma_{2} \sigma_{4}\right) *\left(\sigma_{1}^{2} \sigma_{3}^{2}\right) \\
& \rho^{+}\left(\alpha_{4}^{+}\right)=\rho^{-}\left(\alpha_{2}^{-}\right)=\left(\sigma_{1} \sigma_{3} \sigma_{2} \sigma_{4} \sigma_{1} \sigma_{3}\right) *\left(\sigma_{2}^{2} \sigma_{4}^{2}\right)
\end{aligned}
$$

where $a * b:=a b a^{-1}$ and the strings in the positive (respectively negative) case correspond to the lines $\left(L_{1}^{+}, L_{3}^{+}, L_{2}^{+}, L_{4}^{+}, L_{5}^{+}\right)$, respectively $\left(L_{1}^{-}, L_{4}^{-}, L_{5}^{-}, L_{2}^{-}, L_{3}^{-}\right)$.

Proof. This is straightforward from Figures 1 and 2 and the choice of lexicographic braids.

Remark 4.14. Replacing $\rho^{-}\left(\alpha_{j}^{-}\right)$by $\tau^{-1} \rho^{-}\left(\alpha_{j}^{-}\right) \tau$, where $\tau:=\sigma_{3} \sigma_{4} \sigma_{2} \sigma_{3} \sigma_{2}$, the strings of the braids correspond to the same sets of ordered lines. After this change, we will keep the original notation $\rho^{-}$.

Our goal is to adapt [ACC03, Theorem 1] to the case of ordered horizontal line arrangements. The main difference is that the orbit of an ordered arrangement is given by the action of the pure braid group instead of the whole braid group as is required in the unordered case. Let us fix an ordered horizontal line arrangement $\mathcal{L}$. Let us consider its braid monodromy $\rho: \pi_{1}\left(\mathbb{C} \backslash A_{\mathcal{L}}\right) \rightarrow \mathbb{P}_{n}$. Fixing a geometric basis $\gamma_{1}, \ldots, \gamma_{r}$ of $\pi_{1}\left(\mathbb{C} \backslash A_{\mathcal{L}}\right), r=\# A_{\mathcal{L}}$, the $r$-tuple $\left(\rho\left(\gamma_{1}\right), \ldots, \rho\left(\gamma_{r}\right)\right)$ is said to determine, or represent, the braid monodromy $\rho$. Recall that $\rho$ was defined up to conjugation in $\mathbb{P}_{n}$; this comes from the two choices that have been made: the base point $*$ and the geometric basis.

Definition 4.15. Two elements of $\mathbb{P}_{n}^{r}$ are said to be equivalent if they can be related by simultaneous conjugation in $\mathbb{P}_{n}$ and Hurwitz moves. Recall that the $j$ th Hurwitz move of $\left(\tau_{1}, \ldots, \tau_{r}\right) \in \mathbb{P}_{n}^{r}$ produces

$$
\left(\tau_{1}, \ldots, \tau_{j-1}, \tau_{j+1}, \tau_{j+1} * \tau_{j}, \tau_{j+2}, \ldots, \tau_{r}\right) .
$$

Remark 4.16. Given $\tau:=\left(\tau_{1}, \ldots, \tau_{r}\right) \in \mathbb{P}_{n}^{r}$ it is important to consider its pseudo-Coxeter element $\chi(\tau):=\tau_{r} \cdots \tau_{1} \in \mathbb{P}_{n}$. Note that $\chi(\tau)$ remains invariant by Hurwitz moves.

Lemma 4.17. Under the above notation, let $\left(\rho\left(\gamma_{1}\right), \ldots, \rho\left(\gamma_{r}\right)\right)$ represent the braid monodromy of $\mathcal{L}$. Then $\tau:=\left(\tau_{1}, \ldots, \tau_{r}\right) \in \mathbb{P}_{n}^{r}$ also represents the braid monodromy of $\mathcal{L}$ if and only if $\tau$ is equivalent to $\left(\rho\left(\gamma_{1}\right), \ldots, \rho\left(\gamma_{r}\right)\right)$.

Proof. The ideas involved in the proof of this lemma are classical, see [ACC03].

TheOREM 4.18. Let $\mathcal{L}_{1}, \mathcal{L}_{2} \subset \mathbb{P}^{2}$ be two ordered affine horizontal line arrangements such that their associated fibered projective arrangements have the same combinatorics. Also let $F: \mathbb{P}^{2} \rightarrow \mathbb{P}^{2}$ be an orientation-preserving homeomorphism such that:

(i) $F(P)=P, P$ being the projection point;

(ii) $F\left(\mathcal{L}_{1}\right)=\mathcal{L}_{2}$ respecting ordering and orientations; and

(iii) $F$ respects the vertical lines preserving both ordering and orientations.

Then both ordered affine horizontal line arrangements have equivalent braid monodromies. 


\section{E. Artal Bartolo et al.}

Proof. The proof essentially follows that in [ACC03, Theorem 1].

Theorem 4.19. There is no order-preserving homeomorphism between $\left(\mathbb{P}^{2}, \cup \mathcal{C}^{+}\right)$and $\left(\mathbb{P}^{2}, \cup \mathcal{C}^{-}\right)$.

Proof. We break the proof up into several steps.

SteP 1. There is no homeomorphism between $\left(\mathbb{P}^{2}, \cup \mathcal{C}^{+}\right)$and $\left(\mathbb{P}^{2}, \cup \mathcal{C}^{-}\right)$preserving the ordering and the orientation on both the plane and the lines.

Proof. This result follows from Theorem 4.18. Recall that $\rho^{-}$has been replaced by a conjugate (Remark 4.14). Let us suppose that $\rho^{+}$and $\rho^{-}$are equivalent.

Let $K^{ \pm}$denote the image of $\rho^{ \pm}$in the pure braid group. Let $c^{ \pm}:=\chi\left(\rho^{ \pm}\left(\alpha^{ \pm}\right)\right)$be the pseudoCoxeter elements of

$$
\begin{aligned}
& \rho^{+}\left(\alpha^{+}\right):=\left(\rho^{+}\left(\alpha_{5}^{+}\right), \rho^{+}\left(\alpha_{3}^{+}\right), \rho^{+}\left(\alpha_{2}^{+}\right), \rho^{+}\left(\alpha_{4}^{+}\right)\right) \quad \text { and } \\
& \rho^{-}\left(\alpha^{-}\right):=\left(\rho^{-}\left(\alpha_{3}^{-}\right), \rho^{-}\left(\alpha_{4}^{-}\right), \rho^{-}\left(\alpha_{5}^{-}\right), \rho^{-}\left(\alpha_{2}^{-}\right)\right) ;
\end{aligned}
$$

since $c^{ \pm}$is invariant by Hurwitz moves, one should have that $c^{+}$and $c^{-}$are conjugate in the pure braid group $\mathbb{P}_{5}$. Replacing $\rho^{-}$by the action of an appropriate inner automorphism, one may assume that $c:=c^{+}=c^{-}$. Let $C_{c}$ be the centralizer of $c$ in $\mathbb{P}_{5}$. Since $\rho^{+}\left(\alpha^{+}\right)$and $\rho^{-}\left(\alpha^{-}\right)$are equivalent, $K^{+}$ and $K^{-}$should be conjugate by an element $g \in \mathbb{P}_{5}$ such that $g \chi\left(\rho^{+}\left(\alpha^{+}\right)\right) g^{-1}=\chi\left(\rho^{-}\left(\alpha^{-}\right)\right)$; that is, $g c=c g$, hence $g \in C_{c}$ (note that Hurwitz moves preserve the groups $K^{ \pm}$and the pseudo-Coxeter elements).

Let us consider the Burau representation of $\mathbb{B}_{5}$ into $\mathrm{GL}\left(5 ; \mathbb{Z}\left[t^{ \pm 1}\right]\right)$. Replacing $t$ by $2 \bmod 5$ one obtains a representation $\beta: \mathbb{B}_{5} \rightarrow \mathrm{GL}(5 ; \mathbb{Z} / 5 \mathbb{Z})$. The subgroup $\beta\left(\mathbb{P}_{5}\right)$ has order $58032 \times 10^{6}$. Let us denote $\tilde{C}_{c}:=\beta\left(C_{c}\right)$, and $\tilde{K}^{ \pm}:=\beta\left(K^{ \pm}\right)$. Checking that $\tilde{K}^{+}$and $\tilde{K}^{-}$are not conjugate in $\operatorname{GL}(5 ; \mathbb{Z} / 5 \mathbb{Z})$ by an element in $\tilde{C}_{c}$ is a matter of comparing finite tables; the groups $\tilde{K}^{ \pm}$have order 30000 while $\# \tilde{C}_{c}=115200$. This has been verified using a GAP4 [GAP00] program. Its source can be found in the version of this work in arXiv:math.AG/0307296 or downloaded from the public site http://riemann.unizar.es/geotop/pub/gap-programs/lines/monodromy_groups.

SteP 2. There is no homeomorphism between $\left(\mathbb{P}^{2}, \cup \mathcal{C}^{+}\right)$and $\left(\mathbb{P}^{2}, \cup \mathcal{C}^{-}\right)$preserving the ordering, the orientations on the plane, and reversing the orientations on the lines.

Proof. Composing such a homeomorphism with the complex conjugation (which preserves the orientation of the plane, but reverses the orientations on the lines) one obtains a homeomorphism that is ruled out by Step 1.

For the remaining cases the following lemma is required.

Lemma 4.20. Fix an orientation on $\mathbb{S}^{3}$ and consider $\varphi: \mathbb{S}^{3} \rightarrow \mathbb{S}^{3}$ a homeomorphism, $K_{1}, K_{2}, L_{1}, L_{2} \subset$ $\mathbb{S}^{3}$ oriented knots such that $K_{1} \cap K_{2}=\emptyset$ and $\varphi\left(K_{i}\right)=L_{i}$ (disregarding orientations), $i=1,2$. Let us set $\vartheta$ and $\varepsilon_{i}= \pm 1, i=1,2$, such that

$$
\vartheta:=\left\{\begin{array}{ll}
1 & \text { if } \varphi \text { preserves orientation, } \\
-1 & \text { otherwise, }
\end{array} \quad L_{i}=\varepsilon_{i} K_{i} .\right.
$$

Then, if $\mathcal{L}_{\mathbb{S}^{3}}$ is the linking number, $\mathcal{L}_{\mathbb{S}^{3}}\left(L_{1}, L_{2}\right)=\vartheta \varepsilon_{1} \varepsilon_{2} \mathcal{L}_{\mathbb{S}^{3}}\left(K_{1}, K_{2}\right)$.

Remark 4.21. Note also that, from the well-known relationship between linking and intersection numbers, if two lines intersect at one point and one considers the oriented knots they define on the oriented boundary of a small ball centered at the intersection point, then their linking number equals 1 . 
STEP 3. There is no homeomorphism between $\left(\mathbb{P}^{2}, \cup \mathcal{C}^{+}\right)$and $\left(\mathbb{P}^{2}, \cup \mathcal{C}^{-}\right)$preserving the ordering, the orientation on the plane, and the orientation on at least one line.

Proof. By Step 1 there should be at least one line whose orientation is reversed and, by hypothesis, at least one line whose orientation is preserved. Both lines should of course intersect at one point. Let us consider $K$ and $K^{\prime}$ their links at the intersection point. According to the notation of Lemma 4.20, the hypotheses imply that $\vartheta=1$ and $\varepsilon \varepsilon^{\prime}=-1$, and hence $\mathcal{L}_{\mathbb{S}^{3}}\left(L, L^{\prime}\right)=-1$ where $L$ and $L^{\prime}$ are the links of two lines around their intersection point. This contradicts Remark 4.21.

STEP 4. There is no homeomorphism between $\left(\mathbb{P}^{2}, \cup \mathcal{C}^{+}\right)$and $\left(\mathbb{P}^{2}, \cup \mathcal{C}^{-}\right)$preserving the ordering and reversing the orientation on the plane.

Proof. Since $\mathrm{HC}^{+}>$2, there exist at least two lines whose orientations are simultaneously either preserved or reversed. Consider their links at the intersection point. According to the notation of Lemma 4.20, the hypotheses imply that $\vartheta=-1$ and $\varepsilon \varepsilon^{\prime}=1$, which again contradicts Remark 4.21.

This concludes the result.

\section{A counterexample}

Since the aforementioned line combinatorics $\mathcal{N}$ and $\mathcal{C}$ have a great symmetry, they verify the equalities $\# \mathcal{M}(\mathcal{N})=\# \mathcal{M}(\mathcal{C})=1$ and hence will not provide pairs of arrangements having the same combinatorics but different topologies. In [Ryb98], the problem is solved by juxtaposition of two arrangements with combinatorics of type $\mathcal{N}$. In this work we follow a simpler approach than can be applied in many different ways. For the sake of brevity, we describe only one example.

Let $\mathcal{H}^{ \pm}:=\mathcal{C}^{ \pm} \cup\left\{N^{ \pm}\right\}$be two new arrangements, where $N^{ \pm}$is the line joining the points $L_{3}^{ \pm} \cap L_{5}^{ \pm} \cap M_{4}^{ \pm}=\left[1: 0:-\gamma^{ \pm}\right]$and $L_{2}^{ \pm} \cap M_{2}^{ \pm}=\left[0: 1:-\left(\gamma^{ \pm}+1\right)\right]$. In particular, $N^{ \pm}: \gamma^{ \pm} x+$ $\left(\gamma^{ \pm}+1\right) y+z=0$. Note that these two arrangements have the same combinatorics, say $\mathcal{H}$, since their equations are conjugate in $\mathbb{Q}(\sqrt{5})$. The new abstract line of $\mathcal{H}$ will be denoted by $N$.

Proposition 5.1. The automorphism group of $\mathcal{H}$ is trivial.

Proof. Let $\psi \in \operatorname{Aut}(\mathcal{H})$. Note that $\psi(N) \neq M_{j}, j=1, \ldots, 5$, since $N$ does not pass through the unique point of multiplicity 5 . Also, note that $N$ contains three double points and each $L_{j}$ contains at most two double points. Hence, $\psi(N)=N, \psi(\mathcal{C})=\mathcal{C}$ and therefore $\psi$ induces an automorphism of $\mathcal{C}$. By simple combinatorial arguments, $\psi\left(M_{4}\right)=M_{4}$ and $\psi\left(M_{2}\right)=M_{2}$, therefore $\psi=1_{\mathcal{H}}$.

Corollary 5.2. $\# \mathcal{M}(\mathcal{H})=2$ and $\mathcal{H}^{ \pm}$are representatives of the two elements.

Theorem 5.3. There is no homeomorphism between $\left(\mathbb{P}^{2}, \cup \mathcal{H}^{+}\right)$and $\left(\mathbb{P}^{2}, \cup \mathcal{H}^{-}\right)$.

Proof. By Proposition 5.1, such a homeomorphism would induce the identity on $\mathcal{H}$, hence having to send $N^{+}$to $N^{-}$. Therefore, it would define an order-preserving homeomorphism between $\left(\mathbb{P}^{2}, \cup \mathcal{C}^{+}\right)$ and $\left(\mathbb{P}^{2}, \cup \mathcal{C}^{-}\right)$. The result follows from Theorem 4.19 .

Remark 5.4. An important feature of this pair of arrangements is the fact that they are defined by conjugate equations in $\mathbb{Q}(\sqrt{5})$. This fact implies that they cannot be distinguished by algebraic methods. Note for example that the fundamental groups of $\mathbb{P}^{2} \backslash \bigcup \mathcal{H}^{+}$and $\mathbb{P}^{2} \backslash \bigcup \mathcal{H}^{-}$have isomorphic profinite completions. 


\section{REFERENCES}

ACC03 E. Artal, J. Carmona, and J. I. Cogolludo, Braid monodromy and topology of plane curves, Duke Math. J. 118 (2003), 261-278. MR1980995(2004k:14015).

ACCM05 E. Artal, J. Carmona, J. I. Cogolludo, and M. Marco, Invariants of combinatorial line arrangements and Rybnikov's example, Proc. 12th MSJ-IRI symposium, Adv. Stud. Pure Math. (Math. Soc. Japan, Tokyo), to appear, arXiv:math.AG/0403543.

CS97 D. C. Cohen and A. I. Suciu, The braid monodromy of plane algebraic curves and hyperplane arrangements, Comment. Math. Helv. 72 (1997), 285-315. MR1470093(98f:52012).

GAP00 The GAP Group, Aachen, St. Andrews, GAP - Groups, Algorithms, and Programming, version 4.2 (2000), available at http://www.gap-system.org.

Ryb98 G. Rybnikov, On the fundamental group of the complement of a complex hyperplane arrangement, Preprint (1998), arXiv:math.AG/9805056.

Enrique Artal Bartolo artal@unizar.es

Departamento de Matemáticas, Campus Plaza de San Francisco s/n, E-50009 Zaragoza, Spain

Jorge Carmona Ruber jcarmona@sip.ucm.es

Departamento de Sistemas informáticos y programación, Universidad Complutense, Ciudad Universitaria s/n, E-28040 Madrid, Spain

José Ignacio Cogolludo Agustín jicogo@unizar.es

Departamento de Matemáticas, Campus Plaza de San Francisco s/n, E-50009 Zaragoza, Spain

Miguel Marco Buzunáriz mmarco@unizar.es

Departamento de Matemáticas, Campus Plaza de San Francisco s/n, E-50009 Zaragoza, Spain 